\title{
Reflets
}

Revue d'intervention sociale et communautaire

\section{Interactions des personnes sans abri avec les services de police : profilage social et droits}

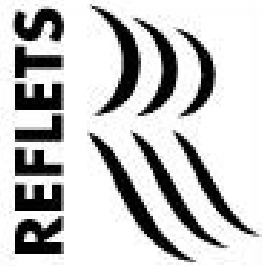

\section{Carol Kauppi, Henri Pallard et Vivan Ellery}

Volume 22, numéro 1, printemps 2016

Profilage social et judiciarisation de la marginalité

URI : https://id.erudit.org/iderudit/1037164ar

DOI : https://doi.org/10.7202/1037164ar

Aller au sommaire du numéro

Éditeur(s)

Reflets, Revue d'intervention sociale et communautaire

ISSN

1203-4576 (imprimé)

1712-8498 (numérique)

Découvrir la revue

Citer cet article

Kauppi, C., Pallard, H. \& Ellery, V. (2016). Interactions des personnes sans abri avec les services de police : profilage social et droits. Reflets, 22(1), 83-122. https://doi.org/10.7202/1037164ar

\section{Résumé de l'article}

Dans le présent article, nous examinons les récits de 29 personnes sans abri afin de comprendre comment leurs interactions avec les policiers présentent des traits de profilage social et racial. Ces personnes sont des femmes et des hommes de 18 à 54 ans provenant du nord-est de l'Ontario. Les personnes autochtones forment la majeure partie de l'échantillonnage (66 \%), tandis que les personnes anglophones, francophones ou membres de groupes racialisés en constituent des parts moins importantes. Les transcriptions des entrevues ont fait l'objet d'une analyse thématique qualitative afin d'identifier des thèmes d'interaction et de les coder. Nous examinons les cinq types d'interaction avec la police les plus communs selon deux aspects : premièrement, comment les personnes sans abri décrivent leurs mauvaises expériences d'interaction du point de vue du profilage social, y compris le profilage racial, et, deuxièmement, comment certaines actions de la police peuvent porter atteinte aux droits des personnes sans abri garantis par les articles 7 à 10 de la Charte canadienne des droits et libertés. Les résultats indiquent que le profilage social est un concept plus pertinent que le profilage racial lorsqu'on considère les expériences vécues par les personnes sans abri, étant donné que la police tend apparemment à cibler l'ensemble des personnes sans abri en leur infligeant un traitement éprouvant, voire violent, qui porte atteinte à leurs droits, notamment ceux garantis par la Charte.
Tous droits réservés @ Reflets, Revue d’intervention sociale et communautaire, 2016
Ce document est protégé par la loi sur le droit d'auteur. L'utilisation des services d'Érudit (y compris la reproduction) est assujettie à sa politique d'utilisation que vous pouvez consulter en ligne. 


\section{Interactions des personnes sans abri avec les services de police : profilage social et droits}

Carol Kauppi

Professeure titulaire, École de service social

Directrice, Centre de recherche en justice et politique sociales,

Université Laurentienne

\section{Henri Pallard}

Professeur titulaire, département de Droit et justice,

Université Laurentienne

\section{Vivan Ellery}

Assistante à la recherche, Centre de recherche en justice et politique sociales, Université Laurentienne

\section{Résumé}

Dans le présent article, nous examinons les récits de 29 personnes sans abri afin de comprendre comment leurs interactions avec les policiers présentent des traits de profilage social et racial. Ces personnes sont des femmes et des hommes de 18 à 54 ans provenant du nord-est de l'Ontario. Les personnes autochtones forment la majeure partie de l'échantillonnage (66\%), tandis que les personnes anglophones, francophones ou membres de groupes racialisés en constituent des parts moins importantes. Les transcriptions des entrevues ont fait l'objet d'une analyse thématique qualitative afin d'identifier des thèmes d'interaction et de les coder. Nous examinons les cinq types d'interaction avec la police les plus communs selon deux aspects : premièrement, comment les personnes sans abri décrivent leurs mauvaises 
expériences d'interaction du point de vue du profilage social, y compris le profilage racial, et, deuxièmement, comment certaines actions de la police peuvent porter atteinte aux droits des personnes sans abri garantis par les articles 7 à 10 de la Charte canadienne des droits et libertés. Les résultats indiquent que le profilage social est un concept plus pertinent que le profilage racial lorsqu'on considère les expériences vécues par les personnes sans abri, étant donné que la police tend apparemment à cibler l'ensemble des personnes sans abri en leur infligeant un traitement éprouvant, voire violent, qui porte atteinte à leurs droits, notamment ceux garantis par la Charte.

Mots clés : profilage racial, profilage social, droits de la personne, contrôle policier, sans-abrisme, nord-est de l'Ontario

\section{Abstract}

This article explores the narratives of 29 homeless people in order to understand how interactions with police officers reflect elements of social and racial profiling. The participants included women and men between the ages of 18 and 54 from northeastern Ontario. Most participants were Indigenous people (66\%) while smaller proportions were Anglophone, Francophone or members of racialized groups. A thematic, qualitative analysis of interview transcripts was conducted to identify and code themes of interactions. We examine the five most common types of interactions with police in two different ways: first, how homeless people described negative interactions with respect to social profiling, including racial profiling, and secondly, how specific actions of police may violate the rights of homeless people under sections 7 to 10 of the Canadian Charter of Rights and Freedoms. The findings suggest that, when considering the experiences of homeless people, social profiling is a more relevant concept than racial profiling as all homeless people appear to be targeted by police and subjected to difficult or even abusive treatment that breaches their human rights as well as rights under the Charter.

Key words: racial profiling, social profiling, human rights, policing, homelessness, northeastern Ontario 


\section{Introduction}

"...nous pouvons définir le profilage social comme toute action menée pour des raisons de sûreté, de sécurité ou de protection du public qui repose sur des stéréotypes fondés sur la pauvreté ou le sans-abrisme, plutôt que sur un soupçon raisonnable, pour cibler une personne et lui faire subir une surveillance accrue ou un traitement particulier. »
Le profilage social se produit quand la police cible davantage des personnes en raison de leur pauvreté ou de leur sans-abrisme, selon O'Grady, Gaetz et Buccieri (2013, p. 542). Cette définition ressemble à celle qu'a adoptée la Commission ontarienne des droits de la personne (CODP, 2003) dans son rapport d'enquête sur le profilage racial, qui donne cette définition assez large du profilage racial :

[...] toute action prise pour des raisons de sûreté, de sécurité ou de protection du public qui repose sur des stéréotypes fondés sur la race, la couleur, l'ethnie, la religion, le lieu d'origine [...] plutôt que sur un soupçon raisonnable, dans le but d'isoler une personne à des fins d'examen ou de traitement particulier. (p. 7)

La CODP ajoute que le profilage racial ${ }^{1}$ peut se fonder sur une combinaison des facteurs mentionnés dans la définition ci-dessus, qui peuvent être en interaction avec d'autres facteurs comme l'âge et le sexe. De même, le profilage selon l'état de santé mentale est décrit comme "toute prise à partie de personnes en fonction non pas de motifs raisonnables, mais de stéréotypes fondés sur la santé mentale ou les dépendances " (CODP, 2014, p. 30). À partir des définitions de la CODP, nous pouvons définir le profilage social comme toute action menée pour des raisons de sûreté, de sécurité ou de protection du public qui repose sur des stéréotypes fondés sur la pauvreté ou le sans-abrisme, plutôt que sur un soupçon raisonnable, pour cibler une personne et lui faire subir une surveillance accrue ou un traitement particulier.

Il est important de tenir compte du concept du profilage social dans le cadre des recherches sur le sans-abrisme, vu les données probantes indiquant une croissance du nombre d'interactions entre les personnes sans abri et la police ces dernières années en dépit de la diminution générale du nombre d'infractions au Code criminel en Ontario (O’Grady, Gaetz et Buccieri, 2013). Depuis 
«Dans la présente étude, nous examinons en profondeur les types d'interaction les plus fréquents entre la police et les personnes en situation de sans-abrisme, ainsi que des thèmes liés au profilage social et racial et aux actions de la police par rapport aux droits garantis par la Charte." les années 1990, un ensemble de facteurs - dont l'augmentation de la pauvreté, l'écart croissant entre les riches et les pauvres, l'insuffisance du logement abordable et les changements apportés au système de sécurité sociale - augmente le risque de sansabrisme parmi la population des personnes ayant une incapacité, des bénéficiaires d'aide sociale et des travailleurs pauvres. Effectivement, la crise économique de 2008 a contribué à une augmentation du taux de sans-abrisme vérifié dans la Ville du Grand Sudbury, lequel a plus que doublé entre 2009 et 2015 (Kauppi, Pallard et Faries, 2015).

Une étude antérieure sur les interactions de la police avec les personnes sans abri avait permis de noter des contacts avec la police qui auraient violé les droits fondamentaux garantis par la Charte canadienne des droits et libertés : notamment, les personnes sans abri ont souvent décrit des contacts avec la police qui comportaient des fouilles et des détentions arbitraires (Kauppi et Pallard, 2009). Dans la présente étude, nous examinons en profondeur les types d'interaction les plus fréquents entre la police et les personnes en situation de sans-abrisme, ainsi que des thèmes liés au profilage social et racial et aux actions de la police par rapport aux droits garantis par la Charte. Étant donné qu'en Ontario, le profilage racial reçoit plus d'attention - par exemple, l'initiative récente de la CODP (2015) qui entend lancer en 2016 un dialogue sur les politiques visant le profilage racial —, il est important d'examiner comment les actions de la police qui sont associées au profilage social et racial des sans-abri peuvent porter atteinte aux droits fondamentaux garantis par la Charte.

La Charte établit les droits et les libertés qui sont le fondement même d'une société libre et démocratique. Toutes les lois du Canada doivent être compatibles avec la Charte; toute loi qui limite les droits établis par la Charte peut être invalidée. Jackman et Porter (2008 et 2014) ont constaté que la Charte comporte un fort potentiel d'utilisation pour assurer aux droits économiques, sociaux et culturels un statut équivalent à un droit constitutionnel. Ils notent toutefois que les observateurs des contentieux fondés sur la Charte ont fait état "d'une certaine timidité » des tribunaux dans les causes qui portent sur le droit d'être "à l'abri du besoin " 
(Jackman et Porter, 2008, p. 228). En effet, à la lumière de leur examen des contestations fondées sur la Charte, ces auteurs notent que " peu de causes ayant trait aux droits socioéconomiques ont été portées devant les tribunaux au cours du premier quart de siècle de la démocratie constitutionnelle au Canada " [notre traduction] (Jackman et Porter, 2008, p. 228).

Cependant, la victoire remportée devant les tribunaux par la Pivot Legal Society en 2015 est significative. Dans l'arrêt Abbotsford c. Shantz, 2015 BCSC 1909, le juge en chef de la Cour suprême de la Colombie-Britannique a conclu que certains règlements municipaux de la Ville d'Abbotsford portent atteinte aux droits des personnes sans abri garantis par l'article 7 de la Charte. Cette victoire confirme les propos de Jackman et Porter (2008), selon lesquels le Canada peut contribuer à la revendication des droits socioéconomiques en démontrant qu'ils sont un aspect essentiel et inhérent des droits de la personne et non des aspects périphériques ou distincts.

Il est important de mener des recherches pour comprendre le lien intrinsèque entre les droits socioéconomiques et les droits garantis par la Charte, car les résultats de telles recherches pourraient alimenter ce débat d'importance vitale à l'échelle nationale et internationale. Par ailleurs, une nouvelle initiative de la CODP visant à combattre le profilage racial en tant que pratique discriminatoire interdite par le Code des droits de la personne de l'Ontario souligne l'intérêt que soulèvent les droits de la personne.

La présente étude se penche sur le lien entre le profilage social et racial et certains droits garantis par la Charte. Cette étude a trois buts :

1) examiner et décrire les types d'interaction avec la police et le système judiciaire à la lumière des récits de personnes sans abri, en portant une attention particulière aux catégories d'interactions les plus souvent décrites;

2) explorer les thèmes liés au profilage social et racial dans les récits des personnes sans abri, en tenant compte particulièrement du sexe et des origines culturelles des participantes et participants;

3) comparer les résultats qui découlent des deuxième et troisième objectifs. 


\section{Méthodologie}

\section{Échantillon}

Dans cette étude, nous avons adopté une définition inclusive et large du sans-abrisme qui englobe les personnes absolument sans abri, anciennement sans abri, logées précairement et à risque d'être sans abri. Une personne absolument sans abri n'a pas d'endroit qu'elle considère sa demeure, ou sa demeure n'est ni une chambre, ni un appartement, ni une maison, ou sa chambre, son appartement ou sa demeure n'est pas la sienne, ou elle habite cet endroit seulement quatre fois par semaine ou moins, ou elle ne passe pas la nuit au même endroit régulièrement. Une personne est à risque de sans-abrisme en raison de sa situation particulière (p. ex. expulsion imminente, revenu extrêmement faible, mauvais traitements dans sa famille, incapacité de payer le loyer, ou condition médicale sans avantages sociaux).

Nous avons contacté plusieurs fournisseurs de services et nous leur avons demandé d'aider au recrutement des participantes et participants, de manière à ce que l'échantillon des personnes sans abri comprenne un large éventail de personnes aux caractéristiques et aux situations variées. À la suite d'un échantillonnage par choix raisonné, nous avons mené des entrevues en janvier et en août 2009 avec 29 participantes et participants dont les circonstances variaient : personnes sans abri pour un temps court ou long; personnes logées dans un refuge, sous une tente ou dans la rue; personnes ayant de piètres conditions en matière de logement. Ces entrevues ont été menées dans les locaux d'organismes de services de première ligne auprès de la population sans abri de Sudbury. Les fournisseurs de services ont facilité les entrevues en aidant au recrutement des participantes et participants, et en prêtant une salle dans leurs bureaux pour la tenue des entrevues. Les protocoles de recherche ont été approuvés par le Comité d'éthique de la recherche de l'Université Laurentienne.

Parmi les 29 participantes et participants, il y avait 12 femmes et 17 hommes âgés de 18 à 54 ans. Plus de la moitié (59\%) était 
des jeunes ou des adultes de 18 à 40 ans. Les deux tiers - 9 femmes et 10 hommes - étaient d'origine autochtone (66\%). Trois étaient francophones et cinq étaient anglophones d'origine européenne. Une personne était d'origine afro-américaine. Par souci de confidentialité, nous avons inclus ce membre d'un groupe racialisé ${ }^{2}$ dans le groupe de personnes autochtones aux fins de l'analyse et de la préparation du rapport de l'enquête. Un autre participant ne connaissait pas ses origines culturelles, car il a été pris en charge par le système de protection de la jeunesse et d'adoption. Bien que nous l'ayons interviewé dans les locaux d'un organisme de services aux Autochtones et qu'il soit le conjoint de fait d'une personne autochtone, nous avons classé ce participant dans la catégorie « autre".

\section{Analyse}

Cette étude fait suite à nos travaux antérieurs qui visaient à reconnaitre la gamme d'expériences décrites par des personnes sans abri qui ont eu des contacts avec les policiers (Kauppi et Pallard, 2009). De leurs réponses, nous avions identifié 16 thèmes portant sur les interactions avec la police ou le système judiciaire. Dans la présente étude, l'éventail des thèmes est plus étendu, de manière à tenir compte de tous les thèmes abordés par les participantes et participants. Nous avons mené une analyse qualitative interprétative afin de mettre au point un système d'organisation des données d'entrevues (Tesch, 1990). La lecture systématique des transcriptions des entrevues a généré une liste plus étendue des thèmes permettant de classer les types de contact avec la police et le système judiciaire. Ces thèmes ont été ensuite appliqués à toutes les sections (unités textuelles) pertinentes des transcriptions des entrevues. Ainsi, nous avons identifié 23 thèmes qui reflètent les termes et expressions employés par les personnes sans abri pour décrire leurs contacts avec la police ou le système judiciaire. Les thèmes visent les politiques, les règlements, les pratiques ou les processus utilisés par les policiers ou le système judiciaire dans leurs interactions avec les personnes sans abri ou presque sans abri. Quatre assistants dans le cadre de cette recherche qualitative ont reçu une formation pour assigner, chacun de leur 
côté, une catégorie aux énoncés des participantes et participants. Les chercheurs ont ensuite examiné les cas de divergence afin de résoudre les différends.

À la deuxième étape de l'analyse, les transcriptions ont fait l'objet d'une analyse plus poussée pour permettre une meilleure compréhension des réactions des personnes sans abri devant les actions des policiers et du sens qu'elles donnent à leurs expériences. Les six catégories liées au harcèlement et aux mauvais traitements subis par la police ont été analysées du point de vue du profilage social (tableau 2).

Tableau 1 :Types d'interaction avec la police portant sur les articles 7, 8, 9 et 10 de la Charte

\begin{tabular}{|l|l|}
\hline \multicolumn{1}{|c|}{ Type d'interaction } & \multicolumn{1}{c|}{ Droits et libertés garantis par la Charte } \\
\hline Obligation de quitter la ville & art. 7. Droit à la vie, à la liberté et à la sécurité de sa personne \\
\hline Fouille et saisie & $\begin{array}{l}\text { art. 8. Droit à la protection contre les fouilles, les perquisitions } \\
\text { ou les saisies abusives }\end{array}$ \\
\hline Détention ou arrestation & $\begin{array}{l}\text { art. 9. Droit à la protection contre la détention ou } \\
\text { l'emprisonnement arbitraires } \\
\text { art. 10. Droits en cas d'arrestation ou de détention }\end{array}$ \\
\hline Exécution excessive des lois & s/o \\
\hline
\end{tabular}

Les comportements de la police touchant les personnes sans abri sont soumis à un ensemble de lois et de règlements qui justifient l'intrusion de la police dans la vie de personnes sans abri. Cette justification est elle-même réglementé par la Charte. À la troisième étape de l'analyse, les thèmes portant sur des types de comportement liés aux droits garantis par les articles 7,8,9 et 10 de la Charte ont été retenus pour une analyse. À ces trois premiers types, nous avons ajouté l'exécution excessive des lois parce qu'elle avait souvent à son origine des allégations de violations de ces droits (tableau 1). Bref, nous avons choisi d'analyser ces quatre thèmes pour deux raisons : ces comportements de la police, premièrement, touchent aux articles 7, 8, 9 et 10 de la Charte et, 
deuxièmement, font intrusion dans la vie des personnes sans abri ou les soumettent à un contrôle particulier.Tous les incidents ont eu lieu en Ontario, surtout dans le nord-est. Nous n'avons pas précisé les lieux des incidents afin de protéger la confidentialité et l'anonymat des participantes et participants.

Le processus d'analyse a tenu compte également du sexe et de l'origine socioculturelle des participantes et participants. Ainsi, leurs témoignages sont accompagnés d'indications précisant leur appartenance à un groupe : Fe pour sexe féminin, Ma pour sexe masculin, Au pour origine raciale autochtone, $\mathrm{Fr}$ pour francophone d'origine européenne, An pour anglophone d'origine européenne et $\mathrm{O}$ pour autre. Par exemple, Fe, Au signale une femme d'origine autochtone. Nous avons aussi attribué des pseudonymes aux participantes et participants. Cette pratique est courante dans la recherche qualitative et vise à humaniser les participantes et participants tout en préservant leur anonymat.

Tableau 2 : Fréquence des types d'interaction mentionnés

\begin{tabular}{|l|c|c|}
\hline \multicolumn{1}{|c|}{ Type d'interaction } & $\begin{array}{c}\text { Fréquence } \\
\text { N }\end{array}$ & $\begin{array}{c}\text { Pourcentage } \\
\%\end{array}$ \\
\hline $\begin{array}{l}\text { 1. Harcèlement, mauvais traitements ou profilage social } \\
\text { a) mauvais traitements physiques } \\
\text { b) mauvais traitements psychologiques } \\
\text { c) provocation } \\
\text { d) abus de pouvoir et de contrôle } \\
\text { e) profilage racial } \\
\text { f) résultats ou effets du profilage social }\end{array}$ & 133 & 39 \\
\hline Droits garantis par la Charte & \multicolumn{2}{|l|}{} \\
\hline 2. Fouille ou saisie & 110 & 32 \\
\hline 3. Détention ou arrestation & 40 & 12 \\
\hline 4. Application excessive des lois & 36 & 10 \\
\hline 5. Obligation de quitter la ville & 25 & 7 \\
\hline
\end{tabular}




\section{Résultats}

\section{Fréquence des types de contact avec la police}

Le tableau 2 indique les cinq types de contact avec la police que les participantes et participants ont mentionnés le plus souvent.En premier lieu figurent le harcèlement ou les mauvais traitements. Nous avons approfondi l'analyse qualitative de ce problème le plus mentionné afin d'examiner la nature des incidents signalés. Les quatre autres thèmes représentant plus de la moitié des incidents mentionnés (61\%) sont traités ensuite. Nous abordons maintenant les thèmes ayant trait au harcèlement, aux mauvais traitements et au profilage social.

\section{Harcèlement, mauvais traitements ou profilage social}

"...les participantes et participants ont décrit les diverses tactiques et relations constituantes du profilage social qui s'exerce à l'endroit des personnes sans abri..."
Collectivement, les participantes et participants ont discuté de harcèlement ou de mauvais traitements dans 133 segments textuels (c'est-à-dire les unités de texte où ces notions sont exprimées). Ce contenu représente $39 \%$ des unités textuelles qui se rapportent aux cinq types de comportement indiqués au tableau 2. Notre analyse porte principalement sur les diverses formes de mauvais traitements et de harcèlement subis par les participantes et participants. Elle tient compte de la façon dont les participantes et participants ont décrit les diverses tactiques et relations constituantes du profilage social qui s'exerce à l'endroit des personnes sans abri :

a) les mauvais traitements physiques;

b) les mauvais traitements psychologiques;

c) la provocation;

d) les abus de pouvoir et de contrôle de façon générale;

e) le profilage racial;

f) les résultats ou effets du profilage social.

Nous reprenons chacune des catégories ci-dessous et tenons à souligner que l'analyse du harcèlement et des mauvais traitements a été menée dans une perspective de profilage social. Les façons spécifiques dont les comportements de la police (décrits dans 
les énoncés des participantes et participants) ont enfreint divers articles de la Charte canadienne des droits et libertés sont présentées à la suite de l'analyse des six catégories.

\section{a. Mauvais traitements physiques}

Les participantes et participants de tous les groupes culturels ont décrit les mauvais traitements physiques qu'ils ont subis lors de leurs contacts avec les policiers. Les personnes autochtones, qui constituaient la majorité (deux tiers) des participantes et participants, ont fourni la plupart des descriptions d'incidents de ce genre. Les personnes anglophones ou francophones et les autres personnes sans abri ont également décrit des expériences de mauvais traitements physiques infligés par les policiers, et certains de ces témoignages sont inclus ci-dessous ${ }^{3}$.

"Elaine, qui était une travailleuse du sexe dans la rue, a décrit au moins trois genres de mauvais traitements physiques et sexuels qu'elle a subis..."
Elaine, qui était une travailleuse du sexe dans la rue, a décrit au moins trois genres de mauvais traitements physiques et sexuels qu'elle a subis :

«Parce qu'ils m'aiment pas, ils s'acharnent contre moi. [Le policier] me tenait, il m'étouffait, tout ça. C'est pas drôle. Tu te sens comme si tu pourras plus jamais respirer.Tu vois,j'étais rendue au point où je [imitant le son de quelqu'un qui manque d'air]. J'arrivais plus à reprendre mon souffle, tu sais. On fait pas ça à quelqu'un. C'est pas correct, ça, vraiment. On fait pas mal à quelqu'un comme ça. J'avais rien fait pour mériter ça.» (Elaine, Fe, An)

«Oui. Un d'eux, ah, il m'a littéralement arraché de la voiture et m'a jetée par terre. Ils m'ont dit d'arrêter de résister, tu sais, mais je résistais même pas. Ils faisaient un «trip de pouvoir», oui.» (Elaine, $\mathrm{Fe}, \mathrm{An}$ )

«Quand j'ai été arrêtée pour prostitution, il y a eu un policier qui a glissé sa main sous ma 
"Des femmes

autochtones ont également décrit les mauvais traitements qu'elles ou leur partenaire ont subis..." chemise et qui a serré mon mamelon, oui. " (Elaine, Fe, An)

Des femmes autochtones ont également décrit les mauvais traitements qu'elles ou leur partenaire ont subis :

"Ils m'ont jetée à terre, ils ont pris ma tête et ils l'ont cognée plusieurs fois contre la voiture. Oui, c'est des vrais trous de cul, excusez mon langage, mais c'est des trous de cul. Il y a beaucoup de brutalité policière, ils font ça aux gens. J'étais enceinte dans ce temps-là. Maintenant, j'ai subi une ligature des trompes. Mais à ce moment-là, j'étais enceinte et j'ai fini par [faire] une fausse couche." (Susan, $\mathrm{Fe}, \mathrm{Au}$ )

"Oui, je veux dire leur attitude, [les policiers] sont des trous de cul, ou bien ils prennent un ton baveux, tu sais ce que je veux dire? Mais à [nom d'une ville de l'Ontario], ils crient après toi, ils sacrent après toi, ils te mettent des menottes avant même de te fouiller. [...] Ils [m']ont suivie, ils ont fait toutes leurs affaires... puis ils m'ont dit de m'étendre par terre et je me suis étendue par terre. Mon mari s'est battu avec eux et, à cause de ça, ils l'ont électrocuté au Taser. Moi, je suis restée étendue par terre pendant qu'il se faisait électrocuter. Ils l'ont traité comme de la marde, ils l'ont brassé un peu, ils lui ont crié après." (Marie, $\mathrm{Fe}, \mathrm{Au}$ )

La dénonciation de Susan sur la fréquence de la brutalité policière se reflète dans les propos d'autres participantes et participants qui racontent qu'on les a étranglés, frappés ou battus, qu'on les a jetés par terre ou assommés, qu'ils ont subi le Taser ou ont été aspergés de poivre de cayenne, ou que des menottes appliquées trop fermement les ont blessés. Le témoignage de Doug ci-dessous indique qu'il a tenté d'éviter d'être malmené en étant docile : «Ils me disent juste de me fermer la gueule et de m'asseoir. 
En tout cas, c'est ce que j'ai fait tout de suite, parce que j'ai déjà été assommé par eux avant, tu comprends?"(Doug, Ma, Au).

Dave explique pourquoi il était inutile d'essayer de s'opposer aux demandes des policiers qui voulaient les fouiller ou de contester les actions des policiers qui exercent une force excessive :

« [J'ai refusé de les laisser fouiller mes possessions.] Six policiers m'ont sauté dessus, ils m'ont électrocuté au Taser, ils m'ont arrosé au gaz poivré. Ils m'ont vraiment bûché dessus, quand même. J'avais la face pleine de sang et ma tête était fendue. Ils m'ont cassé un bras et ma clavicule était fêlée. C'est qu'ils m'ont cogné dessus en maudit, écoute. J'ai essayé de [déposer une plainte], mais, en cour, la police a juste à dire : "Il résistait". Ils disent juste ça et ça suffit. C'est pas mal difficile de gagner contre un policier. » (Dave, Ma, An)

"Les hommes et les femmes de tous les groupes culturels ont discuté d'incidents où la police a utilisé envers eux une force excessive ou de la «brutalité ».»
Les hommes et les femmes de tous les groupes culturels ont discuté d'incidents où la police a utilisé envers eux une force excessive ou de la "brutalité ". Étant sans abri ou ayant vécu diverses formes de sans-abrisme ainsi qu'un certain niveau de contact avec la police et le système judiciaire, tous les participants et participantes croyaient que les policiers les ciblaient en tant que membres d'un groupe spécifique. Ils estimaient que la police les ciblait parce qu'ils étaient des personnes sans abri, des personnes qui vivaient dans la rue - des personnes ayant un casier judiciaire ou des antécédents de maladie mentale ou de dépendance - ou des personnes autochtones.

\section{b. Mauvais traitements psychologiques}

Jeanne et Gilles, des participants francophones, ont discuté de tactiques qui leur semblaient injustes et troublantes.

«Il m'est arrivé qu'on m'arrête et qu'on me demande de vider mes poches. Des fois, je trouve ça injuste qu'ils puissent automatiquement présumer [quelque chose]. » (Jeanne, Fe, Fr) 
«Marie, une

participante

autochtone, a décrit

les insultes qu'elle a

entendues lorsque des policiers s'adressaient à des personnes autochtones..."
« Si tu as un dossier criminel, ils vont souvent être sur ton dos. Ils vont te questionner, ils vont t'achaler. Ils vont te questionner, ils vont te fouiller tout le temps. Ils vont... ah, ils sont méchants. Ils sont méchants, oui, ils sont méchants. » (Gilles, Ma, Fr)

On ne saurait dire si ces deux personnes ont été ciblées à cause de leur statut de francophones minoritaires, de leur vulnérabilité en tant que personnes associées à la communauté des gens de la rue ou, comme Gilles l'affirme, de leur casier judiciaire.

Marie, une participante autochtone, a décrit les insultes qu'elle a entendues lorsque des policiers s'adressaient à des personnes autochtones :

"Mais les gars autochtones, ils [les policiers] les harcèlent vraiment. Franchement, c'est parce que j'ai [l'air] d'une Blanche qu'ils me harcèlent pas tellement. [C'est] juste parce que les policiers sont des trous de cul et qu'eux, ils sont des Autochtones et des sans-abri. Et eux [les policiers], ils les regardent de haut [et disent] "Retourne à ta réserve", tu sais, "Si tu veux vivre comme un animal, va-t'en chez vous". Rien que des insultes stupides. Ça me dérange de les répéter. »(Marie, $\mathrm{Fe}, \mathrm{Au}$ )

En tant que personne aux origines mixtes qui dit qu'on ne la reconnait pas comme une personne autochtone en raison de son apparence, Marie différencie son expérience dans la rue de celle des personnes « racialisées » en raison de leurs traits physiques ou de leur apparence. Susan, une autre femme autochtone, n'a pas bénéficié de la protection qu'offre apparemment le fait de "passer pour une Blanche". "Tu peux pas leur dire non. [Si tu leur dis non], là, ils font tout ce qu'ils veulent. Ils pensent que parce qu'ils sont dans la police, ils peuvent [faire n'importe quoi], tu sais. " (Susan, Fe, Au) 


\section{c. Provocation}

"Les participantes

et participants

autochtones, surtout, ont discuté des comportements

des policiers qui semblaient les inciter à réagir de telle sorte qu'ils aggravent leur situation. Un participant a dit : "Ils essaient de te provoquer »."
Les participantes et participants autochtones, surtout, ont discuté des comportements des policiers qui semblaient les inciter à réagir de telle sorte qu'ils aggravent leur situation. Un participant a dit : "Ils essaient de te provoquer ». Une autre participante a expliqué ce dont elle a été témoin :

"Le policier qui parlait à quelques jeunes autochtones le faisait comme un vrai mal élevé. Il a même sacré. C'est comme s'il voulait juste qu'ils aient une réaction à ce qu'il disait. Il était vraiment effronté et, tu sais, c'est comme s'il les poussait à faire quelque chose par la manière qu'il leur parlait. Et eux, ils voulaient faire quelque chose, mais les deux se sont retenus. " (Linda, $\mathrm{Fe}, \mathrm{Au})$

Certains participants et participantes ont décrit ce qui arriverait s'ils donnaient suite à de telles provocations : on leur infligerait des formes de mauvais traitements physiques, comme ceux qui sont mentionnés ci-dessus, et on les accuserait de résister à une arrestation. Comme Linda l'a noté dans sa description d'un incident dont elle a été témoin lorsqu'elle accompagnait les jeunes autochtones ciblés, ceux-ci "se sont retenus » et n'ont pas réagi au comportement grossier et provocateur du policier. D'autres ont également mentionné être au courant de lutilisation policière de la provocation comme tactique :

«Ah, il voulait juste, il essayait de me pousser à réagir." (Michael, $\mathrm{Ma}, \mathrm{Au}$ )

«Mais peu importe les policiers qu'ils envoient, parfois ils sont baveux et effrontés. Tu sais, quand ils savent ce qu'il faut dire pour te faire réagir. Tu réponds quelque chose et làààààà, tu les as en plein dans la face. J'aime pas ça. Tous ces policiers-là, ils se mettent en plein dans ta face.» (Susan, $\mathrm{Fe}, \mathrm{Au}$ ) 
"Doug a aussi

expliqué que lui et ses amis n'avaient d'autre choix que d'obéir aux policiers, même s'ils croyaient que les policiers n'avaient pas raison de les soupconner. Sa référence aux armes à feu que portent les policiers est une reconnaissance du fait que les policiers ont le pouvoir de donner l'ordre de se plier à leur volonté." "
Michael et Susan ont tenu des propos semblables en ce qui concerne la tactique de provocation pour susciter une réaction. D'autres participantes et participants ont parlé des tactiques du genre «se mettre dans ta face " qu'ils ont vues ou subies.

\section{d. Abus de pouvoir et de contrôle}

Les personnes autochtones ont parlé d'un sentiment d'impuissance devant les actions policières visant à exercer un contrôle sur elles. La Commission ontarienne des droits de la personne ne disait autre chose lorsqu'elle affirmait que «les préoccupations liées au profilage sont en rapport direct avec les notions de pouvoir et d'exercice discrétionnaire du pouvoir " (2003, p. 10).

Wanda a décrit le traitement qu'elle a subi et a expliqué qu'elle avait appris à taire ses opinions, à montrer sa soumission et à craindre la police :

"J'ai appris des choses quand j'ai découvert comment on traite le monde, mais je m'attendais pas que ça aille si loin. Ils ont demandé [de fouiller mon sac à main].J'étais mal placée pour dire non. Ils avaient le pouvoir sur moi.» (Wanda, $\mathrm{Fe}, \mathrm{Au})$

«J'oserais jamais, jamais, jamais, jamais, jamais [faire quoi que ce soit].J'ai trop peur d'eux; c'est comme s'ils avaient une espèce de mission, ils aiment pas qu'une femme ait des opinions, ils aiment pas que n'importe qui ait des opinions. Il fallait que tu sois soumise. " (Wanda, $\mathrm{Fe}, \mathrm{Au}$ )

Doug a aussi expliqué que lui et ses amis n'avaient d'autre choix que d'obéir aux policiers, même s'ils croyaient que les policiers n'avaient pas raison de les soupçonner. Sa référence aux armes à feu que portent les policiers est une reconnaissance du fait que les policiers ont le pouvoir de donner l'ordre de se plier à leur volonté.

"Ils nous disaient de fermer nos crisses de gueules. "Va t'asseoir là-bas." Tu sais, comme 
"Michael, Fred, Paul et Wayne ont fait des commentaires qui correspondent à la définition $d u$ profilage racial élaborée par la Commission ontarienne des droits de la personne (CODP, 2003). Ces participants ont mentionné des préjugés fondés sur l'apparence, le racisme ou le ciblage de personnes autochtones..." un chien. "Va t'asseoir là-bas. Va t'accroupir làbas." On n'avait pas de choix, il fallait le faire. Ils avaient des fusils. Puis ils fouillent toutes nos affaires, notre lit, nos sacs. Il faut que tu les laisses faire. " (Doug, $\mathrm{Ma}, \mathrm{Au}$ )

La comparaison qu'utilise Doug (" comme un chien ») révèle son sentiment que les policiers le traitaient comme s'il était moins qu'un humain. Le sentiment exprimé rejoint le commentaire de Wanda ci-dessus, de même que les commentaires ci-dessous sur les mauvais traitements que subissent les personnes autochtones.

\section{e. Profilage racial}

Dans leurs descriptions de leurs contacts avec la police et le système judiciaire, les participants autochtones ont noté spécifiquement que la "racialisation " était le premier motif de plusieurs actions policières menées contre les personnes autochtones. Michael, Fred, Paul et Wayne ont fait des commentaires qui correspondent à la définition du profilage racial élaborée par la Commission ontarienne des droits de la personne (CODP, 2003). Ces participants ont mentionné des préjugés fondés sur l'apparence, le racisme ou le ciblage de personnes autochtones :

"Mais ce qu'ils font que je trouve intimidant, c'est qu'ils font semblant de venir te prendre. Ils te regardent pour voir comment tu vas réagir, ou ils arrêtent leur voiture d'un coup sec pour voir si tu vas partir en courant. De petites choses stupides comme ça, tu vois? C'est parce qu'ils profilent les gens, pas vrai? Profiler, tu comprends, comme à cause de ton apparence. " (Michael, $\mathrm{Ma}, \mathrm{Au}$ )

«D'habitude, ils présumaient que j'avais fait quelque chose. Ou la moitié du temps, ils le présumaient parce que je ressemblais à la description. Ou [à la description] de quelqu'un 
d'autre. Parce qu'ils présumaient toujours que j'avais des drogues ou une arme de quelque sorte. Et ils me demandaient toujours de vider mes poches. » (Fred, Ma, Au)

"[Les policiers], on dirait, euh, qu'ils ont une dent contre, euh, les Autochtones, donc, les considérer comme racistes... Je me mêlais de mes affaires, puis il y en a deux qui m'ont dit de sortir de la ville. Et je n'avais rien fait. [Ils ont dit] : "Il faut que tu sortes de la ville, va-t'en n'importe où. Retourne à ta réserve, ou va-t'en dans une autre ville. Mais reviens pas ici." » (Paul, $\mathrm{Ma}, \mathrm{Au})$

«Le service de police a décidé, ils ont dit : «Bon, là, on en a assez. "Je suppose qu'ils étaient tannés de voir toute la population autochtone... Ils [les Autochtones] se font harceler ici régulièrement. Je dirais que c'est depuis [le début des années 1990], hum, pour ce qui est de ça. " (Wayne, $\mathrm{Ma}, \mathrm{Au})$

Selon Wayne, les mauvais traitements subis par les personnes autochtones se sont accentués depuis le début des années 1990. Un autre homme autochtone, Leo, mentionne que, lors de sa détention au poste de police, il a entendu plusieurs références aux «maudits Indiens ». Selon la définition de la CODP (2003, p. 7), le profilage racial repose sur des stéréotypes qui engendrent un niveau de surveillance accru ou un traitement différent et est inadmissible, même s'il est subtil ou inconscient. Le traitement que Mandy a subi lorsqu'elle a été détenue par la police était loin d'être subtil, et cela importe peu de savoir si les raisons d'avoir mis en doute sa parole en tant que femme autochtone étaient inconscientes ou pas.

"Quand j'étais enceinte de quatre mois et demi avec des jumeaux, mon ex m'a frappée à coups de poing.J'étais en train de faire une fausse couche, et les policiers ont pensé que je mentais, donc 
ils m'ont accusée de... de ce qu'ils appellent ça quand ils pensent que tu mens. Ils ont dit :"Oh, non, t'es pas enceinte, c'est juste que tu portes pas un tampon." S'ils m'avaient amenée à l'hôpital, mes enfants seraient encore là aujourd'hui. Mais ils m'ont laissée là, à saigner, presque saigner à mort, au poste de police pendant trois heures et demie, avant de finalement dire "Appelez l'ambulance". Les ambulanciers ont même écrit une déclaration qui disait que quand ils sont entrés et qu'ils m'ont vue dans la cellule, j'avais l'air de quelqu'un qui avait été poignardé à mort parce qu'il y avait du sang partout. " (Mandy, $\mathrm{Fe}, \mathrm{Au}$ )

Les expériences vécues par Mandy — la violence conjugale, le refus de la police de croire que son partenaire l'avait agressée, l'accusation d'entrave à la justice, la détention en prison, la fausse couche et la perte de ses jumeaux - l'ont traumatisée. De plus, elle a affirmé que ses droits ont été enfreints constamment et que la police lui a rarement, voire jamais, fourni de l'aide.

\section{f. Résultats ou effets du profilage social}

La CODP (2003) a noté que le profilage est une stratégie inefficiente et inefficace pour la prévention du crime. En outre,

"...le profilage sape la confiance du public envers le travail de la police et contribue à la croissance du cynisme et de la méfiance envers le système judiciaire. " le profilage sape la confiance du public envers le travail de la police et contribue à la croissance du cynisme et de la méfiance envers le système judiciaire. Les participantes et participants de cette étude ont émis des idées qui rejoignent les inquiétudes soulevées par la CODP.

"Moi, j'ai un problème de confiance devant n'importe quelle autorité. J'ai pas confiance en la loi ou en rien d'autre. [C'est] à cause de la marde qu'ils ont fait à mon père, ça fait que... ça a toujours rapport avec la famille, tu sais. " (Ellen, $\mathrm{Fe}, \mathrm{Au}$ ) 
Les expériences familiales communes de profilage racial ou social, les expériences personnelles et le bouche-à-oreille ont transmis la croyance que la police n'est pas digne de confiance. Marie, une femme autochtone, se demandait si elle pouvait se souvenir d'un seul cas où des policiers l'avaient aidée, avant de conclure : «jamais». Elle a dit : "Je n'ai pas grand confiance en eux ». Une autre femme autochtone, Darlene, consciente du fait que la police harcèle les personnes autochtones, ne voulait pas que la police sache où elle demeure. Son attitude pouvait la rendre réticente à appeler les policiers, vu sa méfiance à leur égard, comme l'ont également expliqué d'autres participantes et participants. Certainement, nos participantes et participants, surtout les personnes autochtones, étaient d'avis que les policiers et le système judiciaire sont le problème en ce qui les concerne.

Ces contacts négatifs avec la police ont un effet pervers, comme le révèlent un homme autochtone et une femme francophone. Leo a décrit l'expérience d'épisodes de déjà vu qui minaient sa santé et son bien-être:

"Certains souvenirs me reviennent et, tu sais, ces souvenirs [de la sensation] de peur, c'est comme s'ils revenaient. Puis tout d'un coup, il y a encore autre chose qui me revient et, là, je commence à avoir mal à la tête. » (Leo, Ma, Au)

Son témoignage au sujet de ses réminiscences correspond à un symptôme typique de l'état de stress post-traumatique (Archibald, 2006). Jeanne a expliqué qu'elle refoulait ses émotions pour pouvoir supporter les expériences de la surveillance policière dans les rues :

«Parce que j'ai été élevée dans les rues, j'ai fini par apprendre, tu sais, à juste tourner le dos à certaines choses et à me débrouiller avec ces affaires-là toute seule. N'importe quoi qui arrive, il faut juste que tu l'enfonces jusqu'à tes orteils et pas te laisser énerver par ça.» (Jeanne, Fe, Fr) 
Bien que cette stratégie lui permette de supporter ses expériences sur le vif du moment, le refoulement (ou la suppression) des émotions et des souvenirs peut devenir plus problématique avec le temps. Mattley (2002) a décrit comment les émotions sont essentielles à la constitution du sens et à l'identité individuelle. Le fil des émotions relie le passé de la personne à ses actions dans le moment présent et à ses actions à venir. Selon Mattley (2002,p. 369), la biographie entière d'une personne est reliée au flux de l'émotivité dans un moment présent. Cette interconnexion a des implications en santé mentale et en thérapie, car le travail sur les émotions est reconnu en tant qu'outil important pour la construction des émotions dans le présent et les relations avec d'autres personnes. Archibald (2006) indique que la reconnaissance et l'affrontement des émotions sont au cœur de plusieurs pratiques de guérison.

"Wanda a commenté les effets du profilage racial. Elle a soulevé la question du manque de respect pour la police, alors que le profilage racial existe en tant que pratique appliquée aux personnes autochtones. La question qu'elle pose, "Où est votre humanité??...»
Wanda a commenté les effets du profilage racial. Elle a soulevé la question du manque de respect pour la police, alors que le profilage racial existe en tant que pratique appliquée aux personnes autochtones. La question qu'elle pose, "Où est votre humanité? ", vise l'aspect dénigrant du traitement des personnes autochtones par la police, mais elle semble aussi revendiquer une plus grande part de justice sociale et de traitement équitable dans les pratiques policières.

"Je leur dis : "Vous vous sentez comment, tu sais, comment voulez-vous qu' on respecte votre badge et votre uniforme si vous nous traitez comme ça?" Je veux juste dire : "Vous vous sentez comment?"Vous avez dû suivre un cours d'humanité, non? Elle est où, votre humanité? Elle est où, votre humanité? » (Wanda, $\mathrm{Fe}, \mathrm{Au}$ )

L'analyse des tactiques qui se rapportent au harcèlement et aux mauvais traitements est assez révélatrice des perspectives des personnes sans abri sur des questions liées au profilage et aux contacts avec la police. Dans la prochaine section, nous examinons comment des actions spécifiques des policiers envers les personnes sans abri peuvent enfreindre leurs droits garantis par la Charte. Cette section analyse les catégories 2 à 5 présentées au tableau 2 . 


\section{Droits garantis par la Charte}

Les récits des personnes sans abri de Sudbury nous ont permis de mieux comprendre comment leurs droits garantis par la Charte canadienne des droits et libertés sont traités par les policiers et, en conséquence, comment leur vie est réglée par les actions des policiers. Comme le montre le tableau 2, les personnes qui ont participé à cette étude ont collectivement décrit un nombre significatif d'incidents liés aux contacts avec la police qui, selon les descriptions fournies, paraissent comme de graves violations de ces droits. Les données de nature exploratoire recueillies dans le cadre de cette étude indiquent dans une certaine mesure comment les personnes associées à la communauté des sans-abri ne bénéficient pas toujours des droits garantis par la Charte.

\section{Fouille ou saisie}

"D’après les commentaires des participants Roger, Josh et Elaine, les policiers n'ont pas tenu compte de l'attente raisonnable $d u$ respect de leurs vies privées et des droits établis par l'article 8 de la Charte en menant leurs fouilles et leurs saisies."
L'article 8 de la Charte canadienne des droits et libertés protège les citoyens contre les fouilles et les saisies arbitraires. Ce droit limite les actions de l'État afin de protéger l'attente raisonnable en matière de respect de la vie privée de toute personne. Lorsque cette attente est plus sérieuse, les conditions qui justifient la fouille et la saisie deviennent plus exigeantes. C'est l'attente raisonnable de respect de la vie privée qui détermine l'ampleur d'une fouille juridiquement admissible (Kauppi et Pallard, 2009). Le gouvernement doit prouver, avant de procéder à la fouille et à la saisie, que ses intérêts sont supérieurs à ceux de la personne qui s'attend au respect de sa vie privée, ou que la personne a renoncé au respect de sa vie privée (voir Hunter c. Southam, [1984] 2 RCS 145 et R. c. Mellenthin, [1992] 3 RCS 615.) D'après les commentaires des participants Roger, Josh et Elaine, les policiers n'ont pas tenu compte de l'attente raisonnable du respect de leurs vies privées et des droits établis par l'article 8 de la Charte en menant leurs fouilles et leurs saisies.

«On a passé tout l'avant-midi à mendier... [on en avait] ramassé assez pour trois bouteilles [...] puis, tout d'un coup, on voit un gros Jimmy qui s'arrête, le gars sort et vient nous fouiller, prend 
une des bouteilles [le policier dit] : «Vous autres, vous boirez pas dans le parc aujourd'hui!» [...] commence à la vider, [moi je] dis : "Ah, man!»" (Roger, Ma, O)

"Ouin, ils [les policiers] la vident et ils me redonnent pas la bouteille. » (Josh, Ma, Au)

«Eux [les policiers], ils vont juste, euh, ils me font sortir tout ce que $j$ 'ai dans mes poches et ils vont mettre tout ça sur la valise du char ou quelque chose de même [...] Ils veulent juste trouver quelque chose. Ils le font, c'est tout." (Elaine, $\mathrm{Fe}, \mathrm{An}$ )

Une fouille "raisonnable " est admissible. Généralement, une fouille et une saisie sont "raisonnables " lorsqu'elles sont autorisées par un mandat. Toutefois, une fouille et une saisie sans mandat peuvent être justifiées lorsque les circonstances les rendent nécessaires, bien que la norme à atteindre soit élevée. Premièrement, la fouille ou la saisie doit être juridiquement admissible. Deuxièmement, la police doit avoir un motif raisonnable et probable de croire que l'objet visé par la fouille ou la saisie se trouve à l'endroit où elle pratique la fouille. Enfin, la fouille et la saisie ne doivent pas être menées «de façon abusive " (Kauppi et Pallard, 2009, p. 1; voir R.c. Collins, [1987] 1 RCS 265).

La fouille et la saisie sans mandat sont aussi " raisonnables " si elles sont menées dans le contexte d'une arrestation légitime. Les policiers ont souvent recours à la détention afin d'obtenir des renseignements. Une arrestation est légitime lorsque la police a un motif raisonnable et probable de croire que la personne est soupçonnée d'un crime ou qu'elle allait prochainement en commettre un (voir R.c. Storrey, [1990] 1 RCS 241 et R. c. Mann, 2004 CSC 52). La police peut mener une fouille sur une personne détenue, mais cette fouille doit se limiter à une fouille par palpation. La personne doit avoir été formellement arrêtée avant que la police puisse mener une fouille complète, y compris la recherche de marques laissées par les seringues ou les drogues, 
à moins que la police ait un motif raisonnable de croire que la personne est en possession de drogues (Pivot Legal Society, 2002, p. 18). Une fouille à nu n'est pas une "procédure routinière " et la police devrait y avoir recours seulement lorsqu'elle a un "motif raisonnable » de la croire nécessaire. Dans notre étude, un participant, Roger, a affirmé que la police a mené une fouille à nu publique.

"Ils nous ont fouillés à nu là, dans la rue principale, en plein jour... [Et si tu refuses] ils t'amènent à la station et font leur fouille à nu là. " (Roger, $\mathrm{Ma}, \mathrm{O})$

Une personne ne devrait pas être obligée de se dévêtir devant une personne du sexe opposé ou dans un lieu public (Éducation juridique communautaire Ontario ou CLEO, 2013, p. 18). Dans notre étude, des participants ont décrit des expériences spécifiques de fouilles et de saisies qui paraissent arbitraires dans les circonstances en question :

«Ils m'ont pris ma couverture [...] quand je dormais dehors. Ils m'ont amené en prison, puis ils ont jeté les couvertures. " (Josh, Ma, Au)

\section{Détention ou arrestation}

"Les expériences de détention et/ou d'emprisonnement décrites par les personnes qui ont participé à notre étude semblent porter atteinte à ces droits établis par les articles 9 et 10 de la Charte."
Les articles 9 et 10 de la Charte canadienne des droits et libertés établissent les droits dont bénéficie une personne détenue ou emprisonnée. On ne peut pas détenir ou emprisonner quelqu'un arbitrairement. De plus, cette personne a certains droits, notamment ceux : a) d'être informée des motifs de son arrestation ou de sa détention; b) d'être informée immédiatement de son droit à l'assistance d'un avocat. Les expériences de détention et/ ou d'emprisonnement décrites par les personnes qui ont participé à notre étude semblent porter atteinte à ces droits établis par les articles 9 et 10 de la Charte.

"Des fois, je me réveille et je suis dans une cellule. Dans une cellule. Et je suis habituée d'avoir à me demander, à demander à une police 
“Qu'est-ce que j’ai fait?”. Ils disent : “Oh, rien. Je vais te laisser partir dans quelques heures, là." " (Darlene, Fe, $\mathrm{Au}$ )

«Mais le temps que je passais dans les cellules devenait de plus en plus long, jusqu'à vingt heures une fois. Plus on te ramasse, plus de temps tu vas passer ici en dedans. »(Doug, $\mathrm{Ma}, \mathrm{Au}$ )

Selon certaines des personnes qui ont participé à notre étude, la police ne leur dit pas toujours pourquoi elles sont placées dans une cellule. Le dialogue suivant entre Elaine (E) et l'intervieweur (I) illustre ce problème :

«E :Ben, il faut que tu leur demandes pour qu'ils te disent [pourquoi ils t'arrêtent].

I : Oh, donc ils ne te le disent pas automatiquement.

E : Non.

I : Ils ne te disent pas que tu as le droit de ne rien dire, des choses comme ça?

E : Le droit de ne rien dire? Non, ils te disent juste de fermer ta gueule. »(Elaine, Fe, An)

Au dire des participantes et participants, la police semble utiliser la détention sans motif valable comme une punition et souvent sans porter d'accusation. Afin de se justifier a posteriori, la police peut toujours déposer un constat d'infraction.

\section{Application excessive des lois}

Selon la Pivot Legal Society (2002, p. 2),

Au Canada, toute personne a le droit aux avantages et aux obligations de la loi. Les punitions infligées sélectivement aux personnes les plus pauvres et marginalisées de notre société ont un effet corrosif sur cette valeur 
"L'application excessive des lois amène parfois les policiers à mettre en péril le droit à la vie, à la liberté et à la sécurité. Marie a affirmé qu'on l'a expulsée d'un édifice en plein milieu de la nuit et qu'on l'a laissée dehors en plein hiver. » démocratique importante et elles tendent vers un système d'exécution des lois qui dépend des caprices et des préjugés des policiers et non de la primauté du droit [notre traduction].

La Charte canadienne des droits et libertés s'applique à tous les citoyens canadiens, sans égard à leur rang ou aux circonstances. Pourtant, les résultats de notre étude révèlent que les personnes sans abri pensent que les policiers ne respectent pas leurs droits.

«Oh, nos droits se font violer chaque jour. Chaque seconde. Chaque minute. On regarde ailleurs et nos droits se font violer. " (Mandy, $\mathrm{Fe}, \mathrm{Au}$ )

L'application excessive des lois amène parfois les policiers à mettre en péril le droit à la vie, à la liberté et à la sécurité. Marie a affirmé qu'on l'a expulsée d'un édifice en plein milieu de la nuit et qu'on l'a laissée dehors en plein hiver.
"Il y avait quelques bâtisses qui ont des buanderies. Puis tu y vas pour dormir dans les buanderies, mais tu te faisais toujours jeter dehors. Souvent les polices venaient. On faisait pas de bruit, on buvait pas, on faisait juste dormir. “Toi, reviens ici, tu t'en vas en prison." C'est ça qu'ils [les policiers] disaient. » (Marie, $\mathrm{Fe}, \mathrm{Au}$ )

Un autre participant a témoigné de son expérience d'avoir été obligé de quitter les lieux alors qu'il dormait au Marché des fermiers.

«On m'a jeté dehors du Marché des fermiers à 4 - à 4 ou 5 heures du matin -, on m'a dit de m'en aller. On faisait juste dormir — ils pouvaient bien voir qu'on faisait juste dormir. [Ils ne tiennent pas compte du fait qu'il pleut et que nous avons trouvé un abri.] [Les policiers disent] : "Non, c'est une propriété privée." Ça me semble un peu insignifiant. [Je me dis intérieurement] 
laisse-le dormir, tu sais? Il fait pas mal à personne, tu comprends? C'est pas, c'est pas comme s'il vandalisait la place. » (Roger, Ma, O)

Les personnes qui ont participé à notre étude ont dit qu'elles se sentent souvent ciblées par les policiers.

"Ils vont t'arrêter et ils vont essayer de te harceler et ils vont tout le temps te demander ton nom et te demander tes papiers d'identité et tout, toujours. Ou bien, ils te font une fouille, des choses comme ça. J'ai vu ça plusieurs fois. » (Gilles, Ma, Fr)

"Je fumais une cigarette dehors [devant une épicerie] et eux [les policiers] ont dit que je quêtais, [mais] tout ce que je faisais c'était de fumer dehors et, maudit, ils allaient me fouiller! Ils sont sortis du char et ils disaient, genre, "Oh, arrête de quêter." Moi, je dis :"Je suis juste ici à l'épicerie avec ma mère. Elle fait ses épiceries et je fume une cigarette, c'est tout.Voyons, laissezmoi tranquille." Mais eux, c'est "Oh, non, non.Je sais que t'es en train de quêter." " (Dave, Ma,An)

«...selon les

participantes et participants à notre étude, il semblerait que certains policiers exécutent les lois de façon discrétionnaire et discriminatoire, souvent en se fondant sur des stéréotypes et la "racialisation "..."
Des stéréotypes fondés sur la "racialisation ", le revenu apparent ou le lieu, par exemple, ne constituent pas des "motifs raisonnables " de croire qu'un crime a été commis (Pivot Legal Society, 2002, p. 18). Cependant, selon les participantes et participants à notre étude, il semblerait que certains policiers exécutent les lois de façon discrétionnaire et discriminatoire, souvent en se fondant sur des stéréotypes et la "racialisation ", comme le révèlent les témoignages ci-dessus.

Les participantes et participants à notre étude ont aussi témoigné d'expériences d'application excessive des lois pour des infractions mineures, comme se trouver en état d'ivresse dans un lieu public et dormir dans un lieu public.

"Si tu bois devant eux, comme si tu prends une gorgée devant eux, ou si tu leur tapes trop sur 
les nerfs, alors là, ils vont t'accuser d'ivresse dans un lieu public. » (Linda, $\mathrm{Fe}, \mathrm{Au}$ )

"Ils me gardent dans une cellule pendant quelques heures jusqu'à ce que je dessoûle. [...] Parce qu'ils ne veulent pas me voir flâner autour. » (Josh, Ma, Au)

«J'étais au parc [nom du parc] et j'avais un livre emprunté à la bibliothèque. Puis je lisais et il faisait vraiment, vraiment chaud. Puis je portais mon manteau et j'étais fatiguée et j'ai pensé, je vais juste me pencher la tête et arrêter de lire. Puis je vais juste m'appuyer la face contre le livre et couvrir ma tête avec mon manteau pour prendre un petit somme. Deux polices m'ont dit de sortir du parc. " (Wanda, Fe, Au)

"...d'après les récits des participantes et participants à notre étude, il semblerait que les policiers utilisent des lois qui ciblent le sans-abrisme comme point de départ pour passer à l'utilisation d'une force excessive. "
Dans toutes les situations où le recours à la force est autorisé, l'intensité de la force utilisée par les policiers pour détenir ou arrêter une personne doit toujours se limiter au minimum nécessaire. Les policiers peuvent recourir à la force seulement lorsqu'elle est autorisée; menacer de recourir à la force physique constitue une "intimidation " aux termes du Code criminel du Canada (Pivot Legal Society, 2002, p. 13). Si les policiers utilisent une force excessive, ils sont passibles d'une accusation aux termes de l'article 26 du Code criminel (Pivot Legal Society, 2002, p. 9). Pourtant, d'après les récits des participantes et participants à notre étude, il semblerait que les policiers utilisent des lois qui ciblent le sans-abrisme comme point de départ pour passer à l'utilisation d'une force excessive.

«Un jour, ma sœur s'est soûlée et je suppose qu'elle a manqué d'argent. Elle était avec un groupe qui est entré dans la Banque Royale au centre-ville pour retirer de l'argent. Là,je sais pas qui a été le premier à commencer la chicane et je suppose qu'ils ont sacré, qu'il y a eu un peu de conflit là, donc l'unité tactique des drogues 
est arrivée à la Banque Royale. D'après ce qu'on m'a dit, c'est écrit dans le rapport que ma sœur a littéralement été jetée par terre et battue, tu comprends, tout le groupe a reçu des coups de poing et des coups de pied et un policier a dit : "C'est tout ce que t'as? T'as rien que ça?", tu comprends, comme pour les provoquer. Pourtant, dans la police, t'es pas censé faire ça. " (Wayne, $\mathrm{Ma}, \mathrm{Au}$ )

« [J'ai refusé de les laisser fouiller mes possessions.] Ah, six polices m'ont sauté dessus.J'ai eu le Taser, j'ai eu le gaz au poivre. » (Dave, Ma, An)

Les résultats présentés dans cette section témoignent nettement d'une application excessive des lois, ou d'un abus de pouvoir, visant la population pauvre et sans abri et, par conséquent, d'une violation de leur droit constitutionnel à l'égalité devant la loi et dans l'application de la loi.

\section{Obligation de quitter la ville}

L'expression starlight tour (voyage à la belle étoile) est issue des événements qui ont entouré la mort par hypothermie de Neil Stonechild, un adolescent autochtone qui, en 1990, a été conduit en voiture à l'extérieur des limites de la ville de Saskatoon par deux policiers et abandonné dans la nuit par des températures glaciales, atteignant moins 28 degrés Celsius (Wright, 2004). Le rapport sur cet incident, Report of the Commission of Inquiry Into Matters Relating to the Death of Neil Stonechild (Wright, 2004), a documente les faits saillants : Stonechild a été détenu dans la ville par deux policiers avant minuit le 24 novembre 1990 et il est mort de froid tôt le matin du 25 novembre 1990 dans un champ à l'extérieur de la ville. La police "a mené une enquête superficielle et tout à fait inadéquate sur ce décès » [notre traduction] (Wright, 2004,p. 212). Par la suite, d'autres enquêtes sur des incidents semblables ont mené à des accusations contre deux agents de police de Saskatoon (Wright, 2004). Il semble que le voyage à la belle étoile soit une pratique assez connue (Comack, 2012). 
«Plusieurs

participants ont indiqué que des policiers les ont obligés à quitter la ville dans des circonstances qui paraissent arbitraires... "Ils nous ont réellement ramassés et ils nous ont conduits à trente, quarante miles en dehors de la ville [et ils ont dit] : "Allez, continuez." "(Josh, $M a, A u)$
La pratique qui consiste à cibler des personnes marginalisées, à les conduire à l'extérieur des limites de la ville et à les abandonner n'est pas autorisée par la loi. L'article 7 de la Charte canadienne des droits et libertés stipule que chacun a droit à la vie, à la liberté et à la sécurité de sa personne et qu'on ne peut porter atteinte à ce droit qu'en conformité avec les principes de justice fondamentale. À tout le moins, une "virée à la belle étoile " porte atteinte à la liberté de la personne et met potentiellement sa vie en danger.

Plusieurs participants ont indiqué que des policiers les ont obligés à quitter la ville dans des circonstances qui paraissent arbitraires :

"Ils m'ont conduit jusqu'aux limites de la ville et m’ont dit : "Reviens pas." " (Roger, $\mathrm{Ma}, \mathrm{Au}$ )

"Ils nous ont réellement ramassés et ils nous ont conduits à trente, quarante miles en dehors de la ville [et ils ont dit] : "Allez, continuez." " (Josh, $\mathrm{Ma}, \mathrm{Au}$ )

«Les polices, ils m'ont conduit cinq miles plus loin, puis ils m'ont laissé là pour que je revienne à pied.» (Gilles, Ma, Fr)

Il semble que l'état de sans-abrisme de ces participants ait été à l'origine des incidents. Aucune loi n'autorise les policiers à obliger une personne à quitter la ville. De telles situations semblent constituer des atteintes au droit à la vie et au droit à la liberté garantis par l'article 7 de la Charte, selon les constatations de la cour dans Abbotsford c. Shantz.

\section{Discussion}

\section{Liens entre le profilage social et les manquements aux droits garantis par la Charte}

En recoupant, d'une part, les six tactiques et relations constituantes du profilage social - mauvais traitements physiques, mauvais traitements psychologiques, provocation, abus de pouvoir et de 
contrôle, profilage racial et conséquences ou résultats — et, d'autre part, les quatre types d'atteinte aux droits et libertés garantis par la Charte étudiés (tableau 2), nous pouvons voir les liens entre ces deux approches à l'analyse des droits. Une ou plusieurs des six catégories associées au profilage social peuvent aussi être considérées comme un manquement aux droits garantis par la Charte. L'analyse a été menée en deux temps. D'abord, nous avons examiné les six catégories du profilage social pour y repérer les catégories d'atteinte aux droits et libertés. Puis, inversement, nous avons examiné les catégories d'atteinte aux droits et libertés pour y repérer des cas de profilage social. Ces deux temps de l'analyse ont mené à des résultats semblables, ce qui indique un enchevêtrement de ces deux cas de figure. Il n'est peut-être pas si étonnant que les problèmes de profilage social et racial évoqués par la Commission ontarienne des droits de la personne correspondent également à des manquements à la Charte canadienne des droits et libertés. Néanmoins, cette constatation fournit des informations nouvelles et de nouveaux appuis à l'utilisation de ces deux types d'analyse pour rendre compte des rapports des personnes sans abri en fonction des pratiques policières. De plus, cette constatation démontre que ces agissements policiers sont contraires à certains principes fondamentaux de la Charte.

Afin d'explorer les différences liées au sexe et au groupe culturel, nous avons procédé à une analyse quantitative portant sur le nombre d'interactions ou de contacts pouvant porter atteinte aux droits et libertés notés (voir tableau 2). Le nombre d'interactions a été calculé en fonction du sexe et de l'appartenance au groupe racial. Ce calcul peut fournir une indication approximative de l'étendue de la différence entre le profilage social et le profilage racial. Aux fins de cette analyse, les francophones et les anglophones ont été groupés ensemble, parce que la taille de l'échantillon des deux sous-groupes était petite. La comparaison de ce groupe au groupe de personnes autochtones n'a révélé aucune différence significative entre eux. De même, aucune différence significative n'apparaît du point de vue du sexe. Nous n'avons donc pas présenté ces tableaux ici. Cependant, il faut noter que la taille de l'échantillon entier $(n=29)$ est petite; 
par conséquent, le résultat indiquant l'absence de différences entre les groupes doit être interprété avec prudence. Ce résultat appuie néanmoins les résultats de l'analyse qualitative, qui indiquent que les personnes sans abri anglophones et francophones ont connu les mêmes types de contact avec les policiers que les personnes autochtones.

\section{Profilage social et racial}

"Les résultats de cette étude indiquent que les récits des personnes sans abri portant sur leurs interactions avec la police et le système judiciaire correspondent aux aspects $d u$ profilage racial...»
Les résultats de cette étude indiquent que les récits des personnes sans abri portant sur leurs interactions avec la police et le système judiciaire correspondent aux aspects du profilage racial décrit par la CODP (2003). Toutefois, la CODP souligne l'importance du profilage racial sans fournir beaucoup d'informations sur d'autres formes de profilage, comme le profilage social et le profilage selon la santé mentale. Par exemple, la CODP (2003) cite la conclusion du rapport présenté au Solliciteur général de l'Ontario par le Groupe d'étude sur les relations entre la police et les minorités raciales :

Dans son rapport de 1989, le Groupe d'étude tirait la conclusion suivante : les minorités visibles sont d'avis que les services policiers leur réservent un traitement particulier; [traduction] «Elles ne croient pas que le maintien de l'ordre se fasse équitablement dans leurs milieux et elles ont étayé leur argumentation de telle façon qu'il faut en tenir compte.» Selon le Groupe d'étude, les minorités raciales aspirent à participer au maintien de l'ordre et à la prévention des délits, mais « lorsqu' on leur attribue une propension à l'acte criminel, on leur refuse l'intégration à la vie de la collectivité.» (p. 11)

Les résultats de la présente étude appuient ces affirmations à certains égards. Les caractéristiques de l'échantillon comme tel suggèrent que le profilage racial est un problème : lors du recrutement des participantes et participants pour cette étude sur les sans-abri ayant eu des interactions avec la police, 
la majorité (deux tiers) des personnes qui se sont présentées étaient autochtones. De plus, en tant que groupe, les personnes autochtones ont vécu toutes les formes de harcèlement et toutes les catégories d'incidents que nous avons classées comme manquements aux droits garantis par la Charte. Néanmoins, l'analyse qui visait à déterminer si les personnes autochtones témoignaient d'interactions avec la police plus graves que celles des personnes non autochtones n'a indiqué aucune différence statistiquement significative entre les groupes. Ce dernier résultat suggère que, dans l'étude et l'analyse des expériences vécues par les personnes sans abri, le profilage social est un concept plus pertinent que le profilage racial, étant donné que la police semble cibler toutes les personnes sans abri sans distinction de race et leur infliger un traitement rude, voire abusif, qui porte atteinte à leurs droits garantis par la Charte. Les femmes ont témoigné des mêmes types d'interaction que les hommes, y compris les mauvais traitements physiques.

\section{La Charte canadienne des droits et libertés}

Les travaux de la Pivot Legal Society ont livré des résultats issus tant de la recherche que de la contestation judiciaire. Notamment, en octobre 2015, dans Abbotsford c. Shantz, le juge en chef de la Cour suprême de la Colombie-Britannique a conclu que les règlements municipaux de la Ville d'Abbotsford "violent les droits à la liberté et à la sécurité des personnes sans abri d'Abbotsford qui sont garantis par l'article 7 de la Charte canadienne des droits et libertés " [notre traduction] (Pivot Legal Society, 2015a, p. 1-2). Cette décision vise directement la stratégie municipale et policière qui consistait à cibler des personnes sans abri au moyen de règlements municipaux qui, essentiellement, rendaient leur existence illégale. Dans son document "Media Backgrounder ", la Pivot Legal Society (2015b) a affirmé que les expériences des personnes sans abri dans cette ville de la Colombie-Britannique sont semblables à celles de "plusieurs Canadiens qui n'ont pas de domicile. Au lieu de chercher des solutions au problème du logement, des municipalités partout au pays choisissent de criminaliser divers aspects du sans-abrisme, 
"Quand des personnes en situation de sans-abrisme sont arrêtées en vertu de lois qui criminalisent des comportements comme dormir dehors ou s'asseoir sur un trottoir ou dans un parc, ces personnes sont fichées par la police pour des comportements qui ne sont pas de nature criminelle, mais qui sont simplement un effet secondaire de leur sans-abrisme. " comme le fait Abbotsford, dans un effort de rendre le problème invisible» [notre traduction].

Si le profilage racial fait l'objet de quelques études, peu portent sur le profilage racial et le sans-abrisme dans le contexte canadien (Bellot et Sylvestre, 2012). Les résultats de la présente étude font voir qu'il faut mener d'autres travaux dans ce domaine. La police cible possiblement les personnes sans abri dans d'autres communautés ontariennes de la même manière que celle décrite par nos participantes et participants. Depuis le début des années 1990, la criminalisation du sans-abrisme est une stratégie employée dans plusieurs communautés ontariennes. Des comportements comme s'asseoir, dormir ou mendier dans des lieux publics sont devenus la cible de sanctions, en raison des inquiétudes qu'inspire le nombre, sans cesse croissant, de personnes sans abri dans les lieux publics qui leur servent nécessairement de milieux de vie. L'effet principal de ces lois s'est fait sentir sur les personnes en situation de sans-abrisme (O'Grady, Gaetz et Buccieri, 2013). Quand des personnes en situation de sans-abrisme sont arrêtées en vertu de lois qui criminalisent des comportements comme dormir dehors ou s'asseoir sur un trottoir ou dans un parc, ces personnes sont fichées par la police pour des comportements qui ne sont pas de nature criminelle, mais qui sont simplement un effet secondaire de leur sans-abrisme. Une personne qui a un casier judiciaire a de la difficulté à obtenir un emploi et un logement; ces lois compliquent encore plus les efforts des personnes qui tentent de se sortir de leur situation de sans-abri (Ozdeger, 2011).

La criminalisation n'est pas la solution au problème du sans-abrisme : c'est une stratégie qui ne marche pas. Selon le consensus des chercheurs, cette approche est un expédient qui ignore la dimension plus large des problèmes socioéconomiques qui obligent les gens à vivre dans la rue (Hodulik, 2001). La criminalisation en tant qu'approche au problème du sansabrisme est contre-productive. Les municipalités criminalisent le comportement des personnes en situation de sans-abrisme, mais elles n'offrent pas de solutions de rechange à ces personnes. Plusieurs communautés, particulièrement les villes dans le nord des provinces canadiennes, n'ont pas de refuges ou de logements 
abordables en nombre suffisant pour répondre aux besoins de l'ensemble de leur population sans abri. Certaines personnes n'ont donc pas d'autre choix que de vivre dans la rue, malgré le fait que les activités nécessaires à la survie dans la rue sont sanctionnées.

\section{Conclusion}

"La transformation des problèmes sociaux en problèmes raciaux, imputée aux gouvernements, s'applique aussi bien au contexte canadien."
Le sans-abrisme est un phénomène omniprésent et en croissance (Kauppi, Pallard et Faries, 2015). Pourtant, il devient de plus en plus évident que la réponse policière à ce problème est problématique. Les résultats de notre étude démontrent que, selon les dires des participantes et participants, des policiers canadiens ont violé des droits garantis par la Charte canadienne des droits et libertés lors de leurs interactions avec les personnes sans abri.

Toutefois, les changements souhaitables ne peuvent pas venir seulement des policiers. La criminalisation du travail du sexe, de la dépendance à l'alcool ou aux drogues et des comportements liés au sans-abrisme, comme la mendicité, ne règle pas ces problèmes. "La criminalisation impose à la société des coûts économiques liés aux systèmes carcéral et judiciaire, mais les coûts sociaux sont certainement plus étendus et profonds que cela. Des exemples de coûts sociaux sont la rupture des ménages lorsque les parents sont incarcérés, l'enracinement de la pauvreté et l'escalade des coûts des soins de santé lorsqu'on vise les symptômes plutôt que les causes fondamentales. " [notre traduction] (Pivot Legal Society, 2002, p. 41). Il faut reconnaitre ces problèmes en tant qu'enjeux médicaux et sociaux pour réaliser des progrès et réparer les dommages déjà faits, en permettant aux personnes sans abri et à la police de s'affranchir de ce système de criminalisation défaillant qui les oblige souvent à se considérer comme des ennemis.

L'étude ethnographique de Fassin (2011) sur le travail de la police à Paris, en France, documente la nature internationale des modèles et des pratiques de profilage social et racial. La transformation des problèmes sociaux en problèmes raciaux, imputée aux gouvernements, s'applique aussi bien au contexte canadien. 
Ses conclusions sur les pratiques policières s'appliquent, de façon tout aussi troublante, aux résultats de la présente étude :

C'est par approximation toute la population des cités [... et] l'ensemble de la catégorie des personnes $[\ldots]$ qui sont devenues suffisamment suspectes en tant que telles pour faire l'objet de manière banalisée de contrôles d'identité et de fouilles à corps qui, dans le meilleur des cas, ne donnent lieu qu'à quelques brimades et, quelquefois, dégénèrent en violences physiques. (p. 338-339)

Fassin (2011) explique ainsi le comportement des policiers :

Leur respect de l'autorité, leur obligation de réserve, la protection de leur emploi et, pour certains, leur adhésion idéologique à cette ligne répressive les conduisent toutefois à accepter et à perpétuer ces pratiques sans gloire. (p. 339)

Dans notre étude, nous avons cerné les mêmes thèmes - la violation des droits de la personne par le harcèlement, par le profilage social ou racial, par les fouilles et les contrôles, ainsi que la violence verbale et physique - que Fassin a identifiés.

Une future étude pourrait faire la collecte de données liées spécifiquement au profilage social et aux violations des droits garantis par la Charte. Une étude de ce genre pourrait permettre un examen plus approfondi de la convergence de ces approches visant à mieux comprendre les interactions des personnes sans abri avec la police. De plus, il faudrait mener d'autres recherches visant à documenter les types d'interaction avec la police dans des communautés spécifiques et à comparer d'autres villes où se combinent profilage racial et social. Dans la présente étude, nous n'avons pas nommé les communautés particulières où les incidents ont eu lieu. Ces renseignements sont demeurés confidentiels afin de protéger l'anonymat des participantes et participants, conformément aux exigences du Comité d'éthique de la recherche. Une étude plus vaste ayant un plus grand nombre 
de participantes et participants pourrait atteindre le but de préciser les endroits où les incidents ont eu lieu tout en assurant la confidentialité.

\section{Notes}

1 Selon la CODP (2005), la notion de race est un construit social : « La classification selon la race n'a aucun fondement scientifique légitime. La génétique nous apprend que les caractéristiques physiques et les profils génétiques sont en plus forte corrélation entre les 'races' qu'entre les membres de chacune. On reconnait aujourd'hui que les notions de race sont principalement fonction de processus sociaux qui cherchent à établir des différences entre les groupes, avec le résultat d'en marginaliser certains par rapport à la société.» (p. 12). Voir aussi Ducharme et Eid (2005).Dans les circonstances, le terme "profilage ethnique " nous parait plus propice, car il nous permet de comprendre le triage social en fonction de traits culturels et linguistiques et non en fonction de différences physiques fondées sur la couleur de la peau ou des cheveux (Peterson et Åkerström, 2014).

2 La racialisation est le processus par lequel une société construit théoriquement les races comme réelles, différentes et inégales de façons qui ont un effet sur la vie économique, politique et sociale (Gittens et Cole, 1995).

3 Les énoncés extraits des récits des participantes et participants sont des traductions libres, leurs témoignages ayant été recueillis en anglais. Dans nos traductions, nous avons essayé de reproduire le caractère immédiat de leurs paroles plutôt que de la langue écrite.

\section{Bibliographie}

ARCHIBALD, Linda (2006). "Decolonization and Healing: Indigenous Experiences in the United States, New Zealand, Australia and Greenland ", Ottawa, Aboriginal Healing Foundation, réf. du 6 avril 2016, http://www.ahf.ca/downloads/ibpengweb.pdf

BELLOT, Céline, et Marie-Ève SYLVESTRE (2012). «La judiciarisation des populations itinérantes : pratiques de profilage ", Revue du CREMIS, Vol. 5, № 1, p. 10-15, réf. du 6 avril 2016, http:// www.cremis.ca/revue-du-cremis/recherche/la-judiciarisation-des-populations-itinerantespratiques-de-profilage

COMACK, Elizabeth (2012). Racialized Policing:Aboriginal People's Encounters with the Police, Fernwood Books, Black Point, NS.

COMMISSION ONTARIENNE DES DROITS DE LA PERSONNE (2003). "Un prix trop élevé : les coûts humains du profilage racial », Toronto, CODP, 94 p., réf. du 6 avril 2016, http://www.ohrc.on.ca/sites/default/files/attachments/Paying_the_price\%3A_The_human_ cost_of_racial_profiling_fr.pdf

COMMISSION ONTARIENNE DES DROITS DE LA PERSONNE (2005). "Politique et directives sur le racisme et la discrimination raciale », Toronto, CODP, réf du 6 avril 2016, http://www.ohrc.on.ca/sites/default/files/attachments/Policy_and_guidelines_on_racism_and_ racial_discrimination_fr.pdf 
COMMISSION ONTARIENNE DES DROITS DE LA PERSONNE (2014). «Politique sur la prévention de la discrimination fondée sur les troubles mentaux et les dépendances ", Toronto, CODP, 125 p., réf. du 6 avril 2016, http://www.ohrc.on.ca/sites/default/files/Policy\%20on\%20Preventing\%20discrimination $\% 20$ based $\% 20$ on $\% 20$ mental\%20health\%20disabilities\%20and\%20addictions_FRENCH_ Accessible_0.pdf

COMMISSION ONTARIENNE DES DROITS DE LA PERSONNE (2015). «Vers une nouvelle politique de la CODP concernant le profilage racial ", Toronto, CODP, réf. du 6 avril 2016, http://www.ohrc.on.ca/fr/centre_des_nouvelles/vers-une-nouvelle-politique-de-la-codpconcernant-le-profilage-racial

DUCHARME, Daniel, et Paul EID (2005). "La notion de race dans les sciences et l'imaginaire raciste : la rupture est-elle consommée? ", dans L'Observatoire de la génétique, $\mathrm{N}^{\circ} 24$, septembrenovembre 2005, réf. du 6 avril 2016, http://www.omics-ethics.org/observatoire/cadrages/cadr2005/c_no24_05/c_no24_05_02. html

ÉDUCATION JURIDIQUE COMMUNAUTAIRE ONTARIO (2013). Les pouvoirs de la police : interception, fouille et perquisition, CLEO, Toronto, réf. du 6 avril 2016, http://www.cleo.on.ca/fr/publications/fpolstop

FASSIN, Didier (2011). La Force de l'ordre : une anthropologie de la police des quartiers, Paris, Éditions du Seuil, coll. "La Couleur des idées ", 400 p.

GITTENS, Margaret, et David COLE (1995). Rapport de la Commission sur le racisme systémique dans le système de justice pénale en Ontario, Imprimeur de la Reine pour l'Ontario, Toronto, réf. du 6 avril 2016, http://www.ontla.on.ca/library/repository/mon/25005/185735.pdf

GROUNDS, Adrian (2004). "Psychological Consequences of Wrongful Conviction and Imprisonment ", Revue canadienne de criminologie et justice pénale, Vol. 46, № 2, p. 165-182.

HANEY, Craig (2001). The Psychological Impact of Incarceration: Implications for Post-Prison Adjustment, Office of the Assistant Secretary for Planning and Evaluation, U.S. Department of Health \& Human Services, réf. du 6 avril 2016, http://aspe.hhs.gov/basic-report/psychological-impact-incarceration\#II

HODULIK, Jennifer (2001). "The Drug Court Model as a Response to Broken Windows Criminal Justice for the Homeless Mentally Ill ", The Journal of Criminal Law and Criminology,Vol. 91, № 4, p. 1073-1100, réf. du 6 avril 2016, http://scholarlycommons.law.northwestern.edu/jclc/vol91/iss4/3

JACKMAN, Martha, et Bruce PORTER (2008). "Socio-Economic Rights under the Canadian Charter ", dans M. Langford (dir.), Social Rights Jurisprudence: Emerging Trends in International and Comparative Law, New York, Cambridge University Press, p. 209-229.

JACKMAN, Martha, et Bruce PORTER (2014). Advancing Social Rights in Canada, Toronto, Irwin Law. KAUPPI, Carol, Henri PALLARD et Arshi SHAIKH (2015). " Mental Illnesses and Homelessness: Experiences of Francophone, Anglophone, and Indigenous Persons in Northeastern Ontario ", The International Journal of Interdisciplinary Cultural Studies, Vol. 9, № 3-4, p. 9-19.

KAUPPI, Carol, et Henri PALLARD (2009). "Homeless People and the Police: Unreasonable Searches and Seizures, and Arbitrary Detentions and Arrests ", Conference of the International Journal of Arts and Sciences, Vol. 1, No 6, p. 344-359. 
KAUPPI, Carol, Henri PALLARD et Emily FARIES (2015). Homelessness in Greater Sudbury: 2015 Period Prevalence Count, Report prepared for the City of Greater Sudbury, Centre de recherche en justice et politique sociales, Sudbury, Université Laurentienne, 28 p.

MATTLEY, Christine (2002). «The Temporality of Emotion: Constructing Past Emotions », Symbolic Interaction, Vol. 25, № 3, p. 363-378.

O'GRADY, Bill, Stephen GAETZ et Kristy BUCCIERI (2013). «Tickets... and More Tickets: A Case Study of the Enforcement of the Ontario Safe Streets Act ", Canadian Public Policy, Vol. $39, \mathrm{~N}^{\circ} 4$, p. $541-558$.

OZDEGER, Tulin (2011). Criminalizing Crisis: The Criminalization of Homelessness in U.S. Cities, The National Law Center on Homelessness \& Poverty, Washington, DC, 57 p., réf. du 6 avril 2016, www.nlchp.org/Criminalizing_Crisis

PETERSON, Abby, et Malin ÅKERSTRÖM (2014). «Introduction to the Special Issue «Policing Ethnicity: Between the Rhetoric of Inclusion and the Policies and Practices of Exclusion» ", Social Inclusion, Vol. 2, No 3, p. 1-4.

PIVOT LEGAL SOCIETY (2015a). BC Supreme Court Rules Homeless Have Right to Public Spaces. Summary of the decision, Vancouver, Pivot Legal Society, réf. du 6 avril 2016, http://www.pivotlegal.org/bc_supreme_court_rules_homeless_have_right_to_public_space

PIVOT LEGAL SOCIETY (2015b). Media Backgrounder: Abbotsford Homeless Lawsuit, Vancouver, Pivot Legal Society, réf. du 6 avril 2016, http://www.pivotlegal.org/bc_supreme_court_rules_homeless_have_right_to_public_space

PIVOT LEGAL SOCIETY (2002). To Serve and Protect:A Report on Policing in the Vancouver East Side, Vancouver, Pivot Legal Society, 52 p., réf. du 6 avril 2016, http://www.pivotlegal.org/to_serve_and_protect

REBER, Susanne, et Robert RENAUD (2005). Starlight Tour:The Last, Lonely Night of Neil Stonechild, Toronto, Random House Canada, $427 \mathrm{p}$.

SYLVESTRE, Marie-Ève, et Céline BELLOT (2014). " Challenging Discriminatory and Punitive Responses to Homelessness in Canada ", dans M. Jackman et B. Porter (dirs.), Advancing Social Rights in Canada, Toronto, Irwin Law, réf. du 6 avril 2016, http://homelesshub.ca/resource/challenging-discriminatory-and-punitive-responseshomelessness-canada

TESCH, Renata (1990). Qualitative Research:Analysis Types and Software Tools, New York, Falmer.

WRIGHT, David (2004). Report of the Commission of Inquiry into Matters Relating to the Death of Neil Stonechild, The Honourable Mr. Justice David H. Wright, Commissioner, Regina, Queen's Printer, 380 p., réf. du 6 avril 2016,

http://justice.gov.sk.ca/stonechild/finalreport/default.shtml

\section{Jurisprudence}

Abbotsford c. Shantz, 2015 BCSC 1909 (British Columbia Supreme Court).

Hunter c. Southam, [1984] 2 RCS 145 (Recueil des arrêts de la Cour suprême du Canada).

R. c. Collins, [1987] 1 RCS 265. 
R. c. Mann, 2004 CSC 52, [2004] 3 RCS 59.

R. c. Mellenthin, [1992] 3 RCS 615.

R. c. Storrey, [1990] 1 RCS 241. 